\title{
Oriented graphs of diameter 2
}

\author{
ZOLTÁN FÜREDI \\ Department of Mathematics \\ University of Illinois, Urbana, IL 61801-2975, USA and \\ Mathematical Institute of the Hungarian Academy of Sciences \\ 1364 Budapest, POB 127 \\ z-furedi@math.uiuc.edu and furedi@math-inst.hu \\ Peter Horak and Chandra M. Pareek \\ Department of Mathematics \\ Kuwait University, Safat 13060 , Kuwait \\ HORAK@MATH-1.sci.kuniv.edu.kw \\ XUding ZHU \\ Department of Applied Mathematics \\ National Sun Yat-sen University, Taiwan
}

June 1997. Slightly revised on September 29, 1997.*

\begin{abstract}
Let $f(n)$ be the minimum number of arcs among oriented graphs of order $n$ and diameter 2. Here it is shown for $n>8$ that $(1-o(1)) n \log _{2} n \leq$ $f(n) \leq n \log _{2} n-(3 / 2) n$.
\end{abstract}

*1991 Mathematics Subject Classification. Primary 05C20, 05C35, 05C15; Secondary 05D99 Key words and phrases. Diameter of graphs, digraphs, crossintersecting families This copy was printed on March 7, 2003 (161 o2.tex) 


\section{Oriented chromatic number}

An oriented graph is a digraph without opposite arcs, i.e., every pair of vertices is connected by at most one arc. An oriented colouring of an oriented graph $D$ is a colouring of its vertices so that any colour class is an independent set, moreover for any two colour classes $U$ and $V$, all the arcs between them have the same orientation, i.e., either all the arcs of $D$ go from $U$ to $V$ or all the arcs go from $V$ to $U$. The oriented chromatic number of $D$ is the minimum number of colours in such colourings. For an unoriented graph $G$, the oriented chromatic number, $\chi_{o}(G)$, of $G$ is defined as the maximum oriented chromatic number of the orientations of $G$. The notion of the oriented chromatic number has been introduced by Courcelle [4]. It was noted in [11] that the oriented chromatic number of the complete bipartite graph, $K_{k, k}$, is $2 k$, the order of the graph. In this paper, the following question is studied.

What is the minimum number of edges of a graph $G$ on $n$ vertices with the property $\chi_{o}(G)=n$ ?

Suppose, $x, y$ are vertices of an oriented graph $D$ such that $x, y$ are neither adjacent nor connected by a directed path of length 2 . Then the colouring of $D$ which colours $x, y$ by one colour, and colours every other vertex by a distinct colour is an oriented colouring of $D$. On the other hand, if $x, y$ are adjacent or connected by a directed path of length 2 , then they cannot be coloured by the same colour. Therefore, an oriented graph $D$ has oriented chromatic number $|V(D)|$ if and only if every pair of vertices of $D$ is connected by an arc or by a directed path of length 2 . In other words, for every pair of vertices of $D$, at least one vertex can be reached from the other in one or two steps by walking along the $\operatorname{arcs}$ of $D$.

\section{Graphs of diameter 2}

Let us define the diameter of an oriented graph $D$ to be the least integer $d$, such that every pair of vertices is connected by a directed path of length at most $d$. Therefore, the question in the previous section is equivalent to the problem of determining the minimum number of arcs in an oriented graph of order $n$ and diameter 2. We denote this number by $f(n)$, i.e., $f(n)=\min \{|E(D)|:|D|=$ $n, \operatorname{diam}(D)=2\}$.

In fact, the problem of determining the function $f(n)$ was originally posed by Erdős, Rényi and Sós [6] in 1966 and later by Znám [13] and Dawes and Meijer [5]. For unoriented graphs the answer to the question of determining the minimum number of edges among all graphs of order $n$ and diameter 2 is trivial. 
Such a graph has $n-1$ edges and the star is the only extremal graph. Katona and Szemerédi [10] showed that

$$
\frac{n}{2} \log _{2} \frac{n}{2} \leq f(n) \leq n\left\lceil\log _{2} n\right\rceil .
$$

The main result of this paper is, that $\lim f(n) /\left(n \log _{2} n\right)=1$ for $n \rightarrow \infty$. We also provide a slight improvement of the upper bound.

Theorem 1 For any $n \geq 9$,

$$
(1-o(1)) n \log _{2} n \leq f(n) \leq n \log _{2} n-\frac{3}{2} n .
$$

The corresponding constructions suggest that characterizing the extremal graphs is probably a very difficult problem. At the end of the paper it is shown that our asymptotic result applies also to, $\bar{f}(n)$, the minimum number of edges of oriented graphs of strong diameter 2 .

\section{Recursive constructions}

To show that $f(n) \leq n \log _{2} n-n$ for $n>3$, we consider the following operation: Let $G, H$ be two vertex disjoint oriented graphs and let $v$ be a vertex of $G$. The oriented graph, $G_{v}(H)$, is obtained from $G$ by replacing the vertex $v$ by the graph $H$. More formally, the vertex set of $G_{v}(H)$ is $V(G) \cup V(H)-v$ and the arc $a=(x, y)$ belongs to $G_{v}(H)$ if one of the following holds:

(i) $a$ is an arc in either $G-v$ or $H$,

(ii) $x \in V(H)$, and $y \in V(G)-v$ and $(v, y)$ is an $\operatorname{arc}$ of $G$,

(iii) $x \in V(G)-v$, and $y \in V(H)$ and $(x, v)$ is and arc of $G$.

It is easy to see that $G_{v}(H)$ is an oriented graph of diameter 2, too.

Now, we construct a sequence $\{H(n)\}_{1}^{\infty}$ of oriented graphs of diameter 2, where $H(n)$ is of order $n$. First set $H(1)$ to be a graph consisting of a single vertex and $H(2)$ to be a graph on two vertices and an arc. Let $G$ be an oriented path of length 2 with initial and terminal vertices $u$ and $v$, respectively. For $n>2$, the graph $H(n)$ is obtained from $G$ by first substituting the vertex $u$ by $H\left(\left\lfloor\frac{n-1}{2}\right\rfloor\right)$ and then $v$ by $H\left(\left\lceil\frac{n-1}{2}\right\rceil\right)$. Let $h(n)$ be the number of arcs of $H(n)$. Then $h(n)=h\left(\left\lfloor\frac{n-1}{2}\right\rfloor\right)+h\left(\left\lceil\frac{n-1}{2}\right\rceil\right)+n-1$. It can be easily proved by induction that

$$
h(n)=(n+1) t-2^{t+1}+2
$$


where $t=\left\lfloor\log _{2} n\right\rfloor$. We have that $h(n)-n \log _{2} n=n\left(\log _{2} n-t\right)+\left(2^{t+1}-n\right)-(t+2)$, which is clearly positive for $n \geq 4$. Even more, for large $n$, we have that

$$
f(n) \leq n \log _{2} n-1.913 \ldots n+o(n),
$$

where the number $1.913 \ldots$ stands for $1+\log _{2} e-\log _{2} \log _{2} e$. One can further improve this constant in the upper bound by about another 0.03 observing that $f(5)=5$ (obtained from an oriented cycle), and again applying the recursion $f(n) \leq \min _{i}\{f(i)+f(n-1-i)+n-1\}$. Especially, this yields the upper bound in the Theorem for all $n>8$.

However, all these efforts have no effect on the linearity of our error term.

\section{Lower bound by the method of crossintersect- ing pairs}

Now, we shall prove the lower bound in Theorem 1 . We apply the very same method what Katona and Szemerédi used. Actually, the method of crossintersecting families was rediscovered several times (e.g., Alspach, Ollmann and Reid [2] in 1975). For developments see, e.g., Tuza [12], and the survey [7]. We are able to improve on the result of Katona and Szemerédi by about a factor of 2 by learning more about the structure of extremal oriented graphs, namely, to take into account, that most of the edges of such a graph join a high-degree and a low-degree vertex. Thus we avoid counting the edges twice. (A similar approach was used in [9].)

Let $G=(V, E)$ be an oriented graph on $n$ vertices of diameter 2. Let $d=$ $\left\lceil\left(\log _{2} n\right)^{2}\right\rceil$, and let $A$ be the set of vertices of $G$ of degree less than $d$. Suppose $A=\left\{x_{1}, x_{2}, \cdots, x_{k}\right\}$ and that the vertex $x_{i}$ is adjacent to $d_{i}$ vertices in $V-A$. Let $s=|V-A|$, and assume that $V-A=\left\{v_{1}, v_{2}, \cdots, v_{s}\right\}$. For each vertex $x$ of $A$, we associate a set $U(x)$ of $0-1$-sequences of length $s$ as follows:

$$
U(x)=\left\{\left(a_{1}, a_{2}, \cdots, a_{s}\right): a_{i}=0 \text { if } x v_{i} \in E \text { and } a_{i}=1 \text { if } v_{i} x \in E\right\} .
$$

Then $\left|U\left(x_{i}\right)\right|=2^{s-d_{i}}$, as $a_{i}$ could be either 0 or 1 in case of $x$ is not adjacent to $v_{i}$. Next we show that each $0-1$-sequence of length $s$ can appear in at most $1+d^{2}$ sets $U(x)$. Indeed, if $\mathbf{a}=\left(a_{1}, a_{2}, \cdots, a_{s}\right) \in U(x) \cap U(y)$ then there is no vertex $v \in V-A$ such that $x v, v y \in E(G)$ or $y v, v x \in E(G)$. Since $G$ has diameter 2, we conclude that $x, y$ are either adjacent or connected by a path of length 2 contained in $A$. Since the subgraph of $G$ induced by $A$ has maximum degree $d$, there are at most $d^{2}$ vertices of $A$ that are connected to $x$ by a directed path (of either direction) of length 1 or 2 . Therefore the $0-1$-sequence a appears 
in at most $d^{2}$ other sets $U(y)$. Hence, by taking the sum $\sum_{1 \leq i \leq k}\left|U\left(x_{i}\right)\right|$, each $0-1$-sequence of length $s$ is counted at most $1+d^{2}$ times. It follows that

$$
\sum_{1 \leq i \leq k} 2^{-d_{i}} \leq 1+d^{2}
$$

Let $e=\sum_{1 \leq i \leq k} d_{i}$. Since $f(x)=2^{-x}$ is convex, Jensen's inequality implies that

$$
k 2^{-e / k} \leq 1+d^{2}
$$

Hence $e \geq k \log _{2} k-k \log _{2}\left(1+d^{2}\right)$.

If $|V-A| \geq 2 n / \log _{2} n$ then the number of edges of $G$ is at least $n \log _{2} n$. If $|V-A|<2 n / \log _{2} n$, then $k=|A|>n-\left(2 n / \log _{2} n\right)$. This implies that

$$
|E(G)| \geq e \geq k \log _{2} k-k \log _{2}\left(1+\left(\log _{2} n\right)^{4}\right)=n \log _{2} n-O\left(n \log _{2} \log _{2} n\right) .
$$

\section{$5 \quad$ Strong diameter and other problems}

Strong diameter. Define the strong diameter of a digraph as the least integer $d$ such that for any pair of vertices, $u$ and $v$, there exist two directed paths of length at most $d$, one from $u$ to $v$ and the other from $v$ to $u$. Then one can ask the following question:

What is the minimum number of $\operatorname{arcs}, \bar{f}(n)$, of an oriented graph on $n$ vertices with strong diameter 2 ?

It turns out that $\bar{f}(n)$ and $f(n)$ are asymptotically the same.

Theorem $2 \quad \bar{f}(n)=n \log _{2} n+O\left(n \log _{2} \log _{2} n\right)$.

Proof: The lower bound follows from the fact that $\bar{f}(n) \geq f(n)$. For the upper bound, we construct the digraph $G$ as follows:

Let $A$ be a set of size $2 k$, and let $B$ be a set of size at most $\left(\begin{array}{c}2 k \\ k\end{array}\right)$, where we choose $k$ to be the minimum integer with $n \leq 2 k+2+\left(\begin{array}{c}2 k \\ k\end{array}\right)$. Then the vertex set of $G$ is $V=A \cup B \cup\{x, y\}$ and there is an arc from $x$ to each element of $A$, an arc from each element of $A$ to $y$, an arc from $y$ to each element of $B$, an arc from each element of $B$ to $x$. Moreover associate each vertex $b$ of $B$ a distinct $k$-subset $S(b)$ of $A$. Then put an arc from $b$ to each element of $S(b)$ and an arc from each element of $A-S(b)$ to $b$. It is straightforward to verify that $G$ indeed has strong diameter 2 , and that the number of arcs as it was claimed. (The only extra care we need is, that for every $a, a^{\prime} \in A$ we have to choose an $S(b)$ with 
$a \in S(b)$ but $a^{\prime} \notin S(b)$. This can be done by less than $k(k-1)$ sets, then the rest of the choices for $S(b)$ 's could be arbitrary.)

We believe, though, that the above construction is close to the optimal, and there must be a relatively large gap between $f(n)$ and $\bar{f}(n)$.

Conjecture $1 \quad \bar{f}(n) \geq n \log _{2} n+\left(\frac{1}{2}+o(1)\right) n \log _{2} \log _{2} n$.

Larger diameters. Let $f(n, d)(\bar{f}(n, d))$ denote the minimum number of arcs in a simple $n$-vertex directed graph of diameter at most $d$. For fixed $d>2$, the order of magnitude is only linear in $n$. This and some conjectures of of Znám [13] and Dawes and Meijer [5] are the subject of a forthcoming manuscript [8].

Homomorphisms of edge coloured graphs. Very recently, N. Alon and T. H. Marshall [1] discussed the following problem. A homomorphism of an edge coloured graph $G_{1}=\left(V_{1}, E_{1}\right)$ to another edge coloured graph $G_{2}=\left(V_{2}, E_{2}\right)$ is a mapping $f: V_{1} \rightarrow V_{2}$ such that for every edge $u v$ of $G_{1}, f(u) f(v)$ is an edge of $G_{2}$, and that the colour of the edge $f(u) f(v)$ is the same as that of $u v$. For an edge coloured graph $G$, let $\lambda(G)$ be the minimal order of an edge coloured graph $H$ such that there is a homomorphism of $G$ to $H$. For an uncoloured graph $G$, we may define $\lambda(G, k)$ to be the maximum of $\lambda\left(G^{\prime}\right)$ where $G^{\prime}$ runs over all edge colourings of $G$ by $k$ colours. Given an uncoloured, undirected graph $G$, it is easy to see that $\lambda(G, 1)=\chi(G)$. Now it turns out that $\lambda(G, 2)$ and $\chi_{o}(G)$ are closely related, although they are different. We point out here that the argument in this paper applies to edge coloured graphs with 2 colours. To be precise, one can ask the following question:

What is the minimum number of edges, $g(n)$, of a graph $G$ on $n$ vertices such that $\lambda(G, 2)=n$ ?

Similarly to the case of the oriented chromatic number, this question is equivalent to the question of finding the minimum number of edges of an edge coloured graph with 2 colours such that any pair of vertices are either adjacent or connected by a path of length two whose two edges are coloured by distinct colours. In general $g(n)$ is different from $f(n)$, for example $f(5)=5$ and $g(5)=6$. However, it is straightforward to modify the argument in this paper to show that

$$
(1-o(1)) n \log _{2} n \leq g(n) \leq n \log _{2} n \text {. }
$$

The details are omitted. 


\section{Acknowledgements}

The research of the first author was supported in part by the Hungarian National Science Foundation under grant OTKA 016389, and by a National Security Agency grant No. MDA904-95-H-1045.

The authors are also indebted to Maria Axenovich (Urbana) for helpful comments.

\section{References}

[1] N. Alon and T. H. Marshall, Homomorphisms of edge-coloured graphs and Coxeter groups, manuscript, 1996.

[2] B. Alspach, L. T. Ollmann, and K. B. Reid, Mutually disjoint families of 0-1 sequences, Discrete Math. 12 (1975), 205-209.

[3] C. Berge, Graphs and hypergraphs, North Holland, Amsterdam, 1973.

[4] B. Courcelle, The monadic second order logic of graphs VI: On several representations of graphs by relational structures, Discrete Applied Math. 54 (1994), $117-149$.

[5] R. Dawes and H. Meijer, Arc-minimal digraphs of specified diameter, J. Combin. Math. and Combin. Comput. 1 (1987), 85-96.

[6] P. Erdős, A. Rényi, and V. T. Sós, On a problem of graph theory, Studia Sci. Math. Hungar. 1 (1966), 215-235.

[7] Z Füredi, Matchings and covers in hypergraphs, Graphs and Combin. 4 (1988), 115-206.

[8] Z. Füredi, Minimal directed graphs of specified diameter, in preparation

[9] Z. Füredi, D. Reimer and A. Seress, Hajnal's triangle-free game and extremal graph problems, Congressus Numerantium 82 (1991), 123-128.

[10] G. Katona and E. Szemerédi, On a problem of graph theory, Studia Scientarum Math. Hungarica 2 (1967), 23-28.

[11] A. V. Kostochka, E. Sopena and X. Zhu, Acyclic chromatic numbers of graphs, J. Graph Theory, to appear.

[12] Z. Tuza, Inequalities for two set-systems with prescribed intersections, Graphs and Combin. 3 (1987), 75-80.

[13] S̆. Znám, The minimal number of edges of a directed graph with given diameter, Acta Fac. Rerum Natur. Univ. Comenian. Math. Publ. 24 (1970), 181-185. 